\title{
Estimativa de parâmetros genéticos em progênies de irmãos completos de eucalipto e otimização de seleção
}

\author{
Genetic evaluation of eucalyptus full-sib progenies and \\ optimization of selection
}

\author{
Thales Augusto Pinto Coelho Nogueira ${ }^{1 *}$, Andrei Caíque Pires Nunes ${ }^{2}$, \\ Glêison Augusto Dos Santos' ${ }^{1}$, Elizabete Keiko Takahashi' ${ }^{3}$, \\ Marcos Deon Vilela De Resende ${ }^{4}$ e Isadora Silva Corradi ${ }^{1}$
}

\begin{abstract}
Resumo
O objetivo desse trabalho foi selecionar as melhores progênies e indivíduos de um teste de progênies de irmãos completos de eucalipto com base no tamanho efetivo populacional, endogamia e ganho genético. O delineamento experimental foi de blocos incompletos, contendo 18 progênies, 75 repetições com uma planta por parcela. No mesmo experimento, 388 clones comerciais de $E$. urophylla $\times$ E. grandis foram utilizados como controle. Aos 6 anos de idade, os caracteres avaliados foram diâmetro à altura do peito, altura total, volume, incremento médio anual (IMA) e sobrevivência. As análises foram realizadas com base no procedimento genético-estatístico de modelos mistos via REML/BLUP. Para otimização de seleção foi feita simulação de 30 diferentes cenários, com diferentes tamanhos efetivos populacionais, taxas de endogamia e ganhos acumulados das populações selecionadas corrigidos pela endogamia. As herdabilidades individuais e de média de progênies apresentaram valores de alta magnitude $\left(h^{2} a>0.50\right.$ e $\left.h^{2} \mathrm{mp}>0.80\right)$, indicando alto controle genético para os caracteres avaliados. O coeficiente de determinação dos efeitos da parcela $\left(c^{2}\right.$ parc) apresentou valores baixos para todos os caracteres e a acurácia foi acima de $90 \%$, o que demonstra baixa influência ambiental. A progênie que apresentou o maior valor genético predito foi um híbrido triplo $(E$. dunni $\times E$. grandis $) \times E$. urophylla que apresentou segregação com indivíduos transgressivos potenciais para clonagem ou cruzamentos direcionados. O cenário simulado mais indicado para seleção alcançou um ganho genético de $114,53 \%$ para a variável IMA, com uma taxa de endogamia de 3,92\%. Tais resultados possibilitarão a redução do cruzamento de indivíduos aparentados, maximização dos ganhos genéticos e a transformação do experimento em pomares de sementes por mudas.
\end{abstract}

PALAVRAS-CHAVES: irmãos germanos; endogamia; tamanho efetivo populacional; teste de progênies

\begin{abstract}
The present study aimed to select the best progenies and individuals of an eucalyptus full-sib progeny test based on effective population size and endogamy. The experiment consisted of 7,261 individuals. The experimental design was established in incomplete blocks, containing 18 progenies, 75 replications, 1,800 randomized blocks within the replicates and with a single tree plot. In the same experiment, 388 E. urophylla $\times$ E. grandis different elite clones were used as controls. At 6 years of age, the evaluated characters were diameter at the breast height, total height, tree volume, mean annual increment (MAI) and survival. All analyzes were performed based on the genetic-statistical procedure of mixed models using REML/BLUP. Selection optimization of the population was done through simulation of 30 scenarios in which different effective population sizes, endogamy rates and accumulated gains corrected by inbreeding were obtained. The individual heritabilities and heritabilities of average progenies presented values of high magnitude ( $h^{2} a>50 \%$ and $h^{2} m p>80 \%$ ), indicating high genetic control for the evaluated traits. The plot determination coefficient $\left(c^{2}\right.$ parc $)$ presented low values for all the characters and the accuracy was above $90 \%$, which demonstrates low environmental influence. The progeny with the highest predicted genetic value was a triple hybrid (E. dunni $\times E$. grandis) $\times E$. urophylla that presented potential transgressive segregant individuals for cloning or directed crosses. The more indicated situation reached a genetic gain of $114.53 \%$ for the variable MAl, with an inbreeding rate of $3.92 \%$. These results will enable reduction of related individuals crossing, maximization of genetic gains and the transformation of the experiment in seed orchards.
\end{abstract}

Keywords: full-sib families; endogamy; effective population size; progenies tests

1. Departamento de Engenharia Florestal, Universidade Federal de Viçosa - UFV. Viçosa / MG, Brasil.

2. Universidade Federal do Sul da Bahia - UFSB. Itabuna / BA, Brasil.

3. Celulose Nipo Brasileira S.A. - CENIBRA. Belo Oriente / MG, Brasil.

4. Centro Nacional de Pesquisa de Florestas, Empresa Brasileira de Pesquisa Agropecuária - Embrapa. Colombo / PR, Brasil.

*Autor correspondente: thalesapcnogueira@gmail.com

Sci. For., Piracicaba, v. 47, n. 123, p. 451-462, set. 2019

DOI: doi.org/10.18671/scifor.v47n123.07 
Nogueira et al. - Estimativa de parâmetros genéticos em progênies de irmãos

completos de eucalipto e otimização de seleção

\section{INTRODUÇ̃̃O}

O setor florestal brasileiro mantém atualmente, em regime de produção, cerca de 7,84 milhões de hectares de áreas de florestas plantadas, sendo 73\% do total, representado pelo gênero Eucalyptus (IBÁ, 2017). Os plantios de clones, espécies e híbridos desse gênero são consolidados pela indústria de base florestal como a principal fonte de insumo nos processos de produção de celulose e papel, carvão vegetal, painéis de madeira reconstituída e usinas de tratamento de madeira (SOUZA et al., 2017).

As empresas e instituições de pesquisa vêm trabalhando intensamente com seleção e geração de materiais genéticos mais produtivos, visando à melhoria da qualidade da madeira em razão do seu uso final, uma vez que grandes avanços são possíveis com o melhoramento genético (ROSADO et al., 2013). No Brasil, o melhoramento do eucalipto teve enorme sucesso, contribuindo para expressivo aumento da produtividade nas plantações destinadas a produção de celulose, painéis e carvão vegetal. Esse sucesso foi obtido, principalmente, devido às técnicas utilizadas dentro de programas de melhoramento (PEREIRA et al., 2015).

Dentre as ferramentas utilizadas no programa de melhoramento do eucalipto, a técnica de hibridação artificial é essencial, sendo um dos principais propulsores do crescente aumento de produtividade (ASSIS, 2014). Com base nas características de interesse para o programa, esta técnica permite a obtenção de sementes híbridas para estabelecimento de testes de progênies e a seleção dos melhores genitores e indivíduos. Assim, os ganhos são maximizados e os genótipos superiores desses testes são selecionados para os plantios clonais (SANTOS et al., 2013).

De acordo com Allard (1971), teste de progênies é um procedimento utilizado para testar o valor de um genótipo baseado no comportamento de sua descendência, produzido segundo um sistema definido de reprodução. A partir dos testes de progênies, é possível avaliar parâmetros genéticos essenciais para orientar os sucessivos ciclos de seleção no programa de melhoramento (VENCOVSKY; BARRIGA, 1992).

O teste de progênies de irmãos completos é um método executado, na maioria das vezes, por meio do processo de polinização controlada com o propósito de avaliar o desempenho dos progenitores femininos, masculinos e das progênies, além de permitir afirmar sobre o mérito genético dos indivíduos (RESENDE; HIGA, 1990). Este tipo de avaliação tem sido empregado no melhoramento de eucalipto em diversos trabalhos, como os de Bison et al. (2006; 2009), Hodge et al. (1996), Lima et al. (2011) Reis et al. (2011), Resende e Higa (1994) e Silva, Boralho e Pots (2004)

Para o avanço das populações de melhoramento a partir dos testes de progênies, é necessário manter a variabilidade genética sem reduzir drasticamente os ganhos com seleção (RESENDE, 1999). A representatividade genética de uma população depende do número de indivíduos selecionados por progênie (ESTOPA et al., 2007). Dessa maneira, é necessário efetuar uma otimização de seleção, praticando a seleção entre e dentro de progênies com limitado número de indivíduos por família, com o objetivo de maximizar os ganhos genéticos e minimizar o cruzamento de indivíduos aparentados (WOOLLIAMS et al., 2015).

Apesar da relevância dos estudos de otimização de seleção em Eucalyptus, trabalhos que abordam este tipo de avaliação são escassos na literatura. Desta forma, este estudo teve como objetivo selecionar as melhores progênies e indivíduos de um teste de progênies de irmãos completos de eucalipto com base no tamanho efetivo populacional, ganho e na endogamia.

\section{MATERIAL E MÉTODOS}

\section{Material}

O teste foi implantado no ano de 2003 no município de Sabinópolis/MG, $18^{\circ} 35^{\prime}$ de latitude $\mathrm{S}$ e $42^{\circ} 57^{\prime}$ longitude $\mathrm{W}$, contendo 18 progênies de irmãos completos obtidas de cruzamentos controlados entre diferentes espécies (Tabela 1), desenvolvidas pela empresa Cenibra durante o projeto Genolyptus.

Aos 6 anos, foram mensurados o diâmetro à altura do peito (DAP) e a altura total das árvores. O DAP foi mensurado com o auxílio de uma fita diamétrica e a altura com o relascópio. Para o cálculo do volume da árvore (VOL) foi utilizada a equação de Schumacher e Hall (1933), sendo utilizado um fator de forma de 0,405. 
Tabela 1. Número de indivíduos por progênie presentes em cada tratamento.

Table 1. Number of individuals per progeny in each treatment.

\begin{tabular}{clcc}
\hline $\mathrm{N}^{\circ}$ & \multicolumn{1}{c}{ Espécies/Híbridos } & Tratamentos & Número de Indivíduos \\
\hline 1 & E. camaldulensis $\times$ E. camaldulensis & $\mathrm{C} 1 \times \mathrm{C} 2$ & 384 \\
2 & E. camaldulensis $\times($ E. urophylla $\times$ E. globulus) & $\mathrm{C} 1 \times \mathrm{UGL}$ & 480 \\
3 & (E. dunni $\times$ E. grandis) $\times$ E. camaldulensis & $\mathrm{DG} \times \mathrm{C} 1$ & 600 \\
4 & (E. dunni $\times$ E. grandis) $\times$ E. dunni & $\mathrm{DG} \times \mathrm{D} 2$ & 56 \\
5 & (E. dunni $\times$ E. grandis) $\times$ E. globulus & $\mathrm{DG} \times \mathrm{GL} 2$ & 592 \\
6 & (E. dunni $\times$ E. grandis) $\times$ E. urophylla & $\mathrm{DG} \times \mathrm{U} 2$ & 600 \\
7 & (E. dunni $\times$ E. grandis) $\times($ E. urophylla $\times$ E. globulus) & $\mathrm{DG} \times \mathrm{UGL}$ & 600 \\
8 & E. grandis $\times$ E. globulus & $\mathrm{G} 1 \times \mathrm{GL} 2$ & 438 \\
9 & E. grandis $\times($ E. urophylla $\times$ E. globulus) & $\mathrm{G} 1 \times \mathrm{UGL}$ & 467 \\
10 & E. grandis $\times$ E. globulus & $\mathrm{G} 2 \times \mathrm{GL} 1$ & 40 \\
11 & E. urophylla $\times$ E. camaldulensis & $\mathrm{U} 1 \times \mathrm{C} 2$ & 319 \\
12 & E. urophylla $\times$ E. dunni & $\mathrm{U} 1 \times \mathrm{D} 2$ & 43 \\
13 & E. urophylla $\times$ E. grandis & $\mathrm{U} 1 \times \mathrm{G} 2$ & 94 \\
14 & E. urophylla $\times$ E. globulus & $\mathrm{U} 1 \times \mathrm{GL} 2$ & 489 \\
15 & E. urophylla $\times$ E. urophylla & $\mathrm{U} 1 \times \mathrm{U} 2$ & 480 \\
16 & E. urophylla $\times($ E. urophylla $\times$ E. globulus) & $\mathrm{U} 1 \times \mathrm{UGL}$ & 479 \\
17 & E. urophylla $\times$ E. camaldulensis & $\mathrm{U} 2 \times \mathrm{C} 1$ & 600 \\
18 & E. urophylla $\times$ E. globulus & $\mathrm{U} 2 \times \mathrm{GL} 1$ & 500 \\
& TOTAL & 18 & $\mathbf{7 2 6 1}$
\end{tabular}

\section{Delineamento experimental e modelo estatístico}

O experimento foi estabelecido no delineamento de blocos incompletos, contendo 18 progênies, 75 repetições, 1.800 blocos casualizados dentro das repetições e uma planta por parcela no espaçamento de espaçamento de 3,0 $\times 2,0$. No mesmo experimento, como testemunhas, foram utilizados 388 diferentes clones comerciais de E. urophylla $\times$ E. grandis para comparação do desempenho das progênies com esses materiais.

O modelo estatístico adotado para a análise dos dados e obtenção dos valores genéticos dos indivíduos, das progênies de irmãos completos e dos clones foi dado por:

$$
y=\boldsymbol{X} r+\boldsymbol{Z} g+\boldsymbol{W} b+e
$$

Em que "y" é o vetor de dados, " $\mathrm{r}$ " é o vetor dos efeitos de repetição (assumidos como fixos) somados à média geral, "g" é o vetor dos efeitos genotípicos individuais (assumidos como aleatórios) quando se analisa progênies e vetor dos efeitos genotípicos quando se analisa clones, "b" é o vetor dos efeitos de blocos, e "e" é o vetor de erros ou resíduos (aleatórios). As letras $\mathbf{X}, \mathbf{Z}$ e $\mathbf{W}$ representam as matrizes de incidência para os referidos efeitos. A análise foi realizada utilizando o modelo 147 do software Selegen-Reml/Blup para avaliar progênies e modelo 2 para clones (RESENDE, 2016).

\section{Otimização de seleção}

A partir dos resultados de ordenamento dos melhores indivíduos pelos seus valores genéticos, foi feito o processo de otimização de seleção da população. Neste processo, foram simulados diferentes cenários de seleção (Tabela 2) de forma a maximizar a diversidade genética na população, reduzir a endogamia e maximizar os ganhos genéticos para o próximo ciclo seletivo.

A representatividade genética de uma população de melhoramento de irmãos completos está relacionada ao número de progênies selecionadas (Nf) e do número de indivíduos selecionados por progênies (kf). Esta representatividade pôde ser avaliada via tamanho efetivo populacional ( $\mathrm{Ne}$ ). (RESENDE, 2002).

Para progênies de irmãos completos, com igual número de indivíduos selecionados por progênie, o tamanho efetivo foi dado por:

$$
N e=\frac{2 N_{f} k_{f}}{k_{f}+1}
$$

O coeficiente de endogamia (F) refere-se à taxa de cruzamentos entre indivíduos aparentados presentes em uma população. Quanto maiores os valores de F em uma população alógama, maior a probabilidade de perdas de alelos via deriva genética. O F apresenta uma relação com o $\mathrm{Ne}$, uma vez que: 
Nogueira et al. - Estimativa de parâmetros genéticos em progênies de irmãos

completos de eucalipto e otimização de seleção

Tabela 2. Otimização de seleção por meio da simulação de 30 diferentes cenários com base no número de progênies e o número de indivíduos por progênie.

Table 2. Optimization of selection through the simulation of 30 different scenarios based on the number of progenies and the number of individuals per progeny.

\begin{tabular}{|c|c|c|c|}
\hline Cenários & $\mathrm{N}^{0}$ de progênies & $\mathrm{N}^{\circ}$ de ind/progênie & $\mathrm{N}^{0}$ total da população \\
\hline 1 & 1 & 30 & 30 \\
\hline 2 & 2 & 3 & 6 \\
\hline 3 & 2 & 5 & 10 \\
\hline 4 & 2 & 10 & 20 \\
\hline 5 & 3 & 3 & 9 \\
\hline 6 & 3 & 5 & 15 \\
\hline 7 & 3 & 10 & 30 \\
\hline 8 & 4 & 3 & 12 \\
\hline 9 & 4 & 5 & 20 \\
\hline 10 & 4 & 10 & 40 \\
\hline 11 & 5 & 3 & 15 \\
\hline 12 & 5 & 5 & 25 \\
\hline 13 & 5 & 10 & 50 \\
\hline 14 & 6 & 3 & 18 \\
\hline 15 & 6 & 5 & 30 \\
\hline 16 & 6 & 10 & 60 \\
\hline 17 & 7 & 3 & 21 \\
\hline 18 & 7 & 5 & 35 \\
\hline 19 & 7 & 10 & 70 \\
\hline 20 & 7 & 15 & 105 \\
\hline 21 & 7 & 20 & 140 \\
\hline 22 & 7 & 25 & 175 \\
\hline 23 & 7 & 30 & 210 \\
\hline 24 & 8 & 3 & 24 \\
\hline 25 & 8 & 5 & 40 \\
\hline 26 & 8 & 10 & 80 \\
\hline 27 & 8 & 15 & 120 \\
\hline 28 & 8 & 20 & 160 \\
\hline 29 & 8 & 25 & 200 \\
\hline 30 & 8 & 30 & 240 \\
\hline
\end{tabular}

$$
F=\frac{1}{2 N e}
$$

A otimização de seleção da população foi feita via simulação de 30 cenários (Tabela 2) nos quais diferentes tamanhos efetivos populacionais $(\mathrm{Ne})$, taxas de endogamia (F) e ganhos acumulados das populações selecionadas corrigidos pela endogamia foram obtidos. A seleção foi realizada entre as melhores progênies (com efeito genético positivo) e, posteriormente, a seleção entre indivíduos dentro de cada progênie. Com isso, a otimização consistiu na escolha do cenário em que se maximizou os ganhos genéticos, com valores de F relativamente baixos e alto valor de Ne para o cenário em questão. O ganho calculado via seleção entre progênies e dentro de progênies foi dado por:

$$
\begin{gathered}
\text { Ganho }(\%)=100 \times\left(\frac{M i-M p}{M p}\right) \\
\text { Ganhocorrigido }(\%)=\operatorname{Ganho}(\%)-[\operatorname{Ganho}(\%) \times F]
\end{gathered}
$$

Onde:

$M i=$ média genotípica dos indivíduos selecionados; $M p=$ média genotípica das progênies; $F=$ endogamia .

\section{RESULTADOS E DISCUSSÃO}

\section{Parâmetros genéticos}

As estimativas de herdabilidades individuais no sentido restrito $\left(h^{2} a\right)$ foram altas para DAP, VOL, IMA e SOB, e mediana para ALT, indicando a existência de diferenças genéticas entre as progênies (Tabela 3). Os valores apresentados indicam a possibilidade de se obter ganhos genéticos para esses caracteres por meio da seleção. 
Tabela 3. Análise de deviance para os caracteres diâmetro à altura do peito (DAP), altura (ALT), Volume (VOL), incremento médio anual (IMA) e sobrevivência (SOB) das progênies de Eucalyptus aos 6 anos de idade.

Table 3. Deviance analysis for the traits diameter at breast height (DBH), total height (TH), volume (VOL), mean annual increment (MAI) and survival (SUR) for Eucalyptus progenies at age 6 years.

\begin{tabular}{clcc}
\hline Caráter & Deviance & LRT \\
\hline DAP & Modelo Reduzido & 19373,42 & $1564,41^{* *}$ \\
& Modelo Completo & 17809,01 & $1332,95^{* *}$ \\
ALT & Modelo Reduzido & 20577,53 & $1554,3^{* *}$ \\
& Modelo Completo & 19244,58 & $1554,32^{* *}$ \\
VOL & Modelo Reduzido & $-11879,21$ & 150 \\
& Modelo Completo & $-13433,51$ & 38042,06 \\
IMA & Modelo Reduzido & 36487,74 & $3552,59^{* *}$ \\
\hline
\end{tabular}

LRT: Teste da razão de verossimilhança; Qui-quadrado $\left(\chi^{2}\right)$ tabelado: 6.63, para o nível de significância de I\%(**), respectivamente, com I grau de liberdade.

O coeficiente de herdabilidade $h^{2} a$ para SOB foi maior que para os demais caracteres (Tabela 4). Estimativas de herdabilidade individual entre 0,01 a 0,15 são consideradas baixas, entre 0,15 a 0,50 são medianas e acima de 0,50 são altas (RESENDE, 2002). DAP, ALT, VOL e IMA apresentaram valores de alta magnitude para $h^{2}$ a. Os valores de $h^{2} a$ obtidos foram superiores aos apresentados por Silva, Boralho e Pots (2004), Li et al. (2007), ambos envolvendo irmãos completos. O primeiro encontrou valores entre 0,19 para o caractere DAP e o segundo de 0,11 para o caractere ALT.

Tabela 4. Estimativas de parâmetros genéticos das progênies de Eucalyptus em relação às variáveis diâmetro à altura do peito (DAP, cm), altura (ALT, $m)$, Volume $\left(\mathrm{VOL}, \mathrm{m}^{3}\right)$, incremento médio anual (IMA, $\mathrm{m}^{3}$.ha ${ }^{-1}$.ano) e sobrevivência (SOB) aos 6 anos de idade.

Table 4. Estimates of genetic parameters for the traits diameter at breast height (DBH in $\mathrm{cm})$, total height (TH in $\mathrm{m})$, volume (VOL in $\mathrm{m}^{3}$ ), mean annual increment (MAI in $\mathrm{m}^{3}$. ha-1. $\mathrm{yr}$ ) and survival (SUR) for Eucalyptus progenies at age 6 years.

\begin{tabular}{|c|c|c|c|c|c|}
\hline Parâmetros & DAP & ALT & VOL & IMA & SOB \\
\hline$h^{2} a$ & 0,5461 & 0,4978 & 0,5141 & 0,5141 & 0,8885 \\
\hline$c^{2}$ parc & 0,0044 & 0,0052 & 0,0048 & 0,0048 & 0,0056 \\
\hline$h^{2} m p$ & 0,8601 & 0,8502 & 0,8528 & 0,8528 & 0,9374 \\
\hline Acprog & 0,9274 & 0,9220 & 0,9235 & 0,9235 & 0,9682 \\
\hline$h^{2} a d$ & 0,3779 & 0,3337 & 0,3482 & 0,3483 & 0,8074 \\
\hline$C V g p \%$ & 19,3515 & 14,1365 & 49,0480 & 49,0582 & 61,3755 \\
\hline$C V e^{\%} \%$ & 31,4809 & 24,4726 & 83,1204 & 83,1286 & 68,3031 \\
\hline$C V r$ & 0,6147 & 0,5776 & 0,5901 & 0,5901 & 0,8986 \\
\hline Média geral & 13,2059 & 20,7480 & 0,1536 & 42,6635 & 0,5443 \\
\hline
\end{tabular}

Acprog: acurácia da seleção de progênies, assumindo sobrevivência completa; $h^{2} a$ : herdabilidade individual no sentido restrito; $h^{2} m p$ : herdabilidade da média de progênies, assumindo sobrevivência completa; $h^{2} a d$ : herdabilidade aditiva dentro de parcela; $c^{2}$ parc: coeficiente de determinação dos efeitos de parcela; $C V g p \%$ : coeficiente de variação genotípica entre progênies em porcentagem; $C V e^{\%}$ : coeficiente de variação residual; $C V r$ : coeficiente de variação relativa.

O coeficiente de determinação dos efeitos ambientais entre parcelas ( $c^{2}$ parc $)$ apresentou valores baixos para todos os caracteres analisados (menores que 0,005). O parâmetro $c^{2}$ parc quantifica a variabilidade das parcelas dentro dos blocos, onde estimativas de até $10 \%$ não chegam a interferir na estimativa dos parâmetros genéticos (RESENDE, 1995). Em razão disso, pode-se inferir que ocorreu baixa variação ambiental entre parcelas e que as estimativas dos parâmetros genéticos não tiveram influência.

Valores altos de acurácia (Acprog) indicam que o delineamento experimental foi adequadamente estabelecido e que os resultados são confiáveis. Acprog) se refere à correlação entre o valor genotípico verdadeiro do material analisado e aquele predito (RESENDE, 2002). Os caracteres avaliados apresentaram alta magnitude de acurácia, com todos os valores acima de 0,90 , indicando alta precisão da seleção e possibilidade de obter ganhos genéticos. A acurácia não apresenta relação com o mérito genético dos indivíduos, o qual deve ser mensurado via valor genético, mas sim com a confiança na seleção dos mesmos. 
Nogueira et al. - Estimativa de parâmetros genéticos em progênies de irmãos

completos de eucalipto e otimização de seleção

O coeficiente de variação relativa $(C V r)$, empregado na avaliação genotípica, apresentou valores inferiores a 1 para todos os caracteres. Resende e Duarte (2007) relatam que é possível obter altos valores de acurácia mesmo com $\mathrm{CVr}^{\prime}$ 's baixos. Segundo esses autores, com 30 a 40 repetições para caracteres de herdabilidade moderada é possível obter acurácias acima de 90\%, mesmo quando a relação $\mathrm{CVgp} / \mathrm{CVe}$ ( $\mathrm{CVr}$ ) é inferior a 0,40. Portanto, valores de $\mathrm{CVr}$ devem ser inferidos em conjunto com o número de repetições (75).

O coeficiente de herdabilidade em nível de média de progênies $\left(h^{2} m p\right)$ foi alto para todos os caracteres (acima de 0,70) (Tabela 4). Isso indica forte controle genético nos caracteres avaliados, além de um alto potencial para seleção entre progênies. Resultados similares para $h^{2} m p$ podem ser encontrados nos estudos de Miranda et al. (2015) e Pinto et al. (2014), apontando que a seleção entre progênies é eficiente e pode alterar o valor da média geral significativamente.

\section{Seleção de potenciais genitores e clones}

Após a análise dos componentes de variância, foi obtido o ranqueamento dos 20 melhores indivíduos potenciais genitores com valores genéticos aditivos preditos $(\mathrm{u}+\mathrm{a})$ superiores para a variável IMA aos 6 anos. Todos os 20 indivíduos pertencem à uma única progênie um híbrido triplo (E. dunni $\times$ E. grandis $\times$ E. urophylla) e apresentaram ganhos significativos (acima de $190 \%$ ) em relação à média geral das progênies com a realização da seleção (Tabela 5).

Tabela 5. Seleção dos 20 melhores indivíduos potenciais genitores de Eucalyptus para a variável IMA ( $\mathrm{m}^{3}$.ha $\mathrm{h}^{-1}$.ano) aos 6 anos de idade.

Table 5. Selection of 20 best Eucalyptus individuals with potential to be genitors for the trait MAI ( $\left.\mathrm{m}^{3} \cdot \mathrm{ha}^{-1} \cdot \mathrm{yr}\right)$ at age 6 years.

\begin{tabular}{|c|c|c|c|c|c|}
\hline Ranking & Bloco & Progênie & Indivíduo & $u+a$ & Gisp (\%) \\
\hline 1 & 16 & $\mathrm{DG} \times \mathrm{U} 2$ & 2238 & 145,9463 & 242,0867 \\
\hline 2 & 4 & $\mathrm{DG} \times \mathrm{U} 2$ & 2138 & 142,2013 & 233,3088 \\
\hline 3 & 47 & $\mathrm{DG} \times \mathrm{U} 2$ & 2483 & 138,6551 & 224,9967 \\
\hline 4 & 26 & $\mathrm{DG} \times \mathrm{U} 2$ & 2313 & 138,5224 & 224,6857 \\
\hline 5 & 34 & $\mathrm{DG} \times \mathrm{U} 2$ & 2381 & 137,5333 & 222,3673 \\
\hline 6 & 52 & $\mathrm{DG} \times \mathrm{U} 2$ & 2526 & 137,0888 & 221,3255 \\
\hline 7 & 26 & $\mathrm{DG} \times \mathrm{U} 2$ & 2319 & 132,3980 & 210,3306 \\
\hline 8 & 27 & $\mathrm{DG} \times \mathrm{U} 2$ & 2323 & 131,3761 & 207,9353 \\
\hline 9 & 34 & $\mathrm{DG} \times \mathrm{U} 2$ & 2378 & 128,5327 & 201,2706 \\
\hline 10 & 7 & $\mathrm{DG} \times \mathrm{U} 2$ & 2161 & 128,2599 & 200,6312 \\
\hline 11 & 53 & $\mathrm{DG} \times \mathrm{U} 2$ & 2533 & 127,8762 & 199,7318 \\
\hline 12 & 32 & $\mathrm{DG} \times \mathrm{U} 2$ & 2361 & 127,6094 & 199,1065 \\
\hline 13 & 49 & $\mathrm{DG} \times \mathrm{U} 2$ & 2502 & 125,2333 & 193,5371 \\
\hline 14 & 34 & $\mathrm{DG} \times \mathrm{U} 2$ & 2383 & 125,1646 & 193,3761 \\
\hline 15 & 46 & $\mathrm{DG} \times \mathrm{U} 2$ & 2475 & 125,1024 & 193,2303 \\
\hline 16 & 47 & $\mathrm{DG} \times \mathrm{U} 2$ & 2485 & 124,5909 & 192,0314 \\
\hline 17 & 21 & $D G \times U 2$ & 2273 & 124,3620 & 191,4948 \\
\hline 18 & 33 & $\mathrm{DG} \times \mathrm{U} 2$ & 2375 & 124,3617 & 191,4941 \\
\hline 19 & 8 & $\mathrm{DG} \times \mathrm{U} 2$ & 2173 & 124,1062 & 190,8953 \\
\hline 20 & 12 & $\mathrm{DG} \times \mathrm{U} 2$ & 2201 & 124,0014 & 190,6496 \\
\hline
\end{tabular}

Gisp (\%): ganho individual de seleção em relação à média geral das progênies $(42,6635)$ em porcentagem; u + a: valor genético aditivo predito.

Conforme Santos et al. (2013), os materiais genéticos produzidos por cruzamentos triplos ou "three-way-cross" podem apresentar maior estabilidade e adaptabilidade ao ambiente. Provavelmente, a superioridade da progênie $\mathrm{DG} \times \mathrm{U} 2$ é resultado da complementaridade entre as espécies/indivíduos, o que pode criar melhores condições adaptativas às condições ambientais variáveis (ASSIS; MAFIA, 2007; SANTOS et al., 2016). Para o melhoramento genético, quanto mais conjuntos gênicos de interesse um genótipo possuir, maior será o potencial de reação do mesmo às mudanças ambientais, gradientes de fertilidade e reações a patógenos, em virtude de sua ampla variabilidade genética e heterozigosidade.

O maior número de indivíduos presentes na progênie $\mathrm{DG} \times \mathrm{U} 2$ pode ter ocasionado um aumento da probabilidade de aparecerem mais indivíduos transgressivos, atribuindo maior predominância 
à esta progênie. Contudo, a seleção massal de indivíduos da mesma progênie pode resultar no cruzamento de indivíduos aparentados, ou seja, é imprescindível a realização da otimização para reduzir efeitos da endogamia sem diminuir drasticamente os ganhos (KAGEYAMA; VENCOVSKY, 1983; ODA; VENCOVSKY, 1989).

Com base no valor genotípico predito, os 20 melhores indivíduos potenciais clones, para a variável IMA, foram ranqueados e comparados com a testemunha. O tratamento testemunha (clone comercial VCP36) apresentou o maior valor genotípico (Tabela 6).

Tabela 6. Seleção dos 20 melhores indivíduos potenciais clones de Eucalyptus para a variável IMA ( $\mathrm{m}^{3}$.ha-1 ${ }^{-1}$ ano) aos 6 anos de idade.

Table 6. Selection of the 20 best Eucalyptus individuals with potential to be clones for the trait MAI $\left(m^{3} \cdot h a^{-1} \cdot y r\right) a t$ age 6 years.

\begin{tabular}{|c|c|c|c|c|c|c|}
\hline Ranking & Bloco & Progênie & Indivíduo & $u+g$ & Gisp (\%) & Gist (\%) \\
\hline 1 & 16 & $\mathrm{DG} \times \mathrm{U} 2$ & 2238 & 167,8722 & 293,4793 & 22,1473 \\
\hline 2 & 4 & $D G \times U 2$ & 2138 & 162,6292 & 281,1902 & 18,3324 \\
\hline 3 & 47 & $\mathrm{DG} \times \mathrm{U} 2$ & 2483 & 157,6646 & 269,5535 & 14,7201 \\
\hline 4 & 26 & $D G \times U 2$ & 2313 & 157,4787 & 269,1178 & 14,5848 \\
\hline 5 & 34 & $D G \times U 2$ & 2381 & 156,0940 & 265,8722 & 13,5773 \\
\hline 6 & 52 & $\mathrm{DG} \times \mathrm{U} 2$ & 2526 & 155,4717 & 264,4135 & 13,1245 \\
\hline 7 & 26 & $D G \times U 2$ & 2319 & 148,9046 & 249,0208 & 8,3461 \\
\hline 8 & 27 & $D G \times U 2$ & 2323 & 147,4740 & 245,6676 & 7,3052 \\
\hline 9 & 34 & $D G \times U 2$ & 2378 & 143,4932 & 236,3369 & 4,4087 \\
\hline 10 & 7 & $\mathrm{DG} \times \mathrm{U} 2$ & 2161 & 143,1113 & 235,4417 & 4,1308 \\
\hline 11 & 53 & $D G \times U 2$ & 2533 & 142,5741 & 234,1826 & 3,7399 \\
\hline 12 & 32 & $D G \times U 2$ & 2361 & 142,2006 & 233,3071 & 3,4681 \\
\hline 13 & 49 & $\mathrm{DG} \times \mathrm{U} 2$ & 2502 & 138,8741 & 225,5101 & 1,0477 \\
\hline 14 & 34 & $D G \times U 2$ & 2383 & 138,7779 & 225,2846 & 0,9777 \\
\hline 15 & 46 & $D G \times U 2$ & 2475 & 138,6908 & 225,0804 & 0,9143 \\
\hline 16 & 19 & $\mathrm{U} 1 \times \mathrm{U} 2$ & 5351 & 138,0524 & 223,5841 & 0,4498 \\
\hline 17 & 47 & $D G \times U 2$ & 2485 & 137,9747 & 223,4019 & 0,3933 \\
\hline 18 & 4 & $\mathrm{U} 1 \times \mathrm{U} 2$ & 5227 & 137,8295 & 223,0616 & 0,2876 \\
\hline 19 & 21 & $\mathrm{DG} \times \mathrm{U} 2$ & 2273 & 137,6542 & 222,6507 & 0,1601 \\
\hline 20 & 33 & $\mathrm{DG} \times \mathrm{U} 2$ & 2375 & 137,6539 & 222,6500 & 0,1599 \\
\hline
\end{tabular}

Gisp (\%): ganho individual de seleção em relação à média geral das progênies $(42,6635)$ em porcentagem; Gisp (\%): ganho individual de seleção em relação à melhor testemunha - clone com maior valor genético $(137,4342)$; u + g: valor genético predito.

Assim, como no ordenamento para potenciais genitores, observou-se a presença de indivíduos pertencentes à progênie $\mathrm{DG} \times \mathrm{U} 2 \mathrm{em}$ grande maioria, com exceção de apenas dois da progênie U1×U2. Na situação apresentada, apenas 20 indivíduos apresentaram desempenho superior ao melhor clone comercial utilizado como testemunha. Os materiais genéticos utilizados como testemunhas apresentam alto grau de melhoramento e são clones comerciais. Segundo Nunes et al. (2016) e Santos et al. (2013), um dos grandes desafios dos programas de melhoramento é superar os clones comerciais plantados atualmente. Dessa maneira, a ocorrência de muitos indivíduos superiores aos clones não era esperada, pois, em virtude dos diferentes níveis de melhoramento dos materiais testados, os clones tendem a apresentar maior produtividade.

\section{Seleção das progênies}

As 18 progênies foram ranqueadas conforme o valor genético predito para a variável IMA. O ganho de seleção por progênie foi calculado utilizando o valor genético em razão da média geral das progênies (Tabela 7).

As 7 primeiras progênies do ranking apresentaram valores maiores que a média geral das progênies, com a $1^{\circ}$ (DGxU2) manifestando valores superiores em $40 \%$ com relação à segunda (U1xU2). O cruzamento triplo ou "three-way-cross" está presente em três das sete melhores progênies apresentadas. A utilização de indivíduos híbridos superiores em novos cruzamentos é recomendada, uma vez que a superioridade genotípica apresentada pelos indivíduos tem origem heterótica. (RESENDE; HIGA, 1990). 
Nogueira et al. - Estimativa de parâmetros genéticos em progênies de irmãos

completos de eucalipto e otimização de seleção

Tabela 7. Seleção das melhores progênies de Eucalyptus, aos 6 anos de idade, para a variável IMA ( $\left.m^{3} \cdot h^{-1} \cdot a n o\right)$.

Table 7. Selection of the best Eucalyptus progenies for the trait MAI ( $m^{3}$.ha-1.1.yr), at age 6 years.

\begin{tabular}{|c|c|c|c|c|}
\hline Ranking & Progênie & $\mathrm{u}+\mathrm{g}$ & Acurácia & Gsp (\%) \\
\hline 1 & $\mathrm{DG} \times \mathrm{U} 2$ & 91,1314 & 0,9667 & 113,6049 \\
\hline 2 & $\mathrm{U} 1 \times \mathrm{U} 2$ & 74,4591 & 0,9662 & 74,5263 \\
\hline 3 & $\mathrm{DG} \times \mathrm{C} 1$ & 65,9318 & 0,9666 & 54,5390 \\
\hline 4 & $\mathrm{U} 1 \times \mathrm{G} 2$ & 54,2586 & 0,9345 & 27,1779 \\
\hline 5 & $\mathrm{DG} \times \mathrm{UGL}$ & 52,6674 & 0,9659 & 23,4483 \\
\hline 6 & $\mathrm{U} 2 \times \mathrm{C} 1$ & 48,4947 & 0,9667 & 13,6678 \\
\hline 7 & $\mathrm{U} 1 \times \mathrm{C} 2$ & 44,6240 & 0,9627 & 4,5952 \\
\hline 8 & $\mathrm{DG} \times \mathrm{D} 2$ & 41,3514 & 0,6294 & $-3,0755$ \\
\hline 9 & U1×D2 & 40,2814 & 0,7438 & $-5,5835$ \\
\hline 10 & U1×UGL & 39,7413 & 0,9634 & $-6,8495$ \\
\hline 11 & G1×UGL & 38,6545 & 0,9638 & $-9,3969$ \\
\hline 12 & $\mathrm{U} 2 \times \mathrm{GL} 1$ & 34,2208 & 0,9589 & $-19,7891$ \\
\hline 13 & $\mathrm{G} 1 \times \mathrm{GL} 2$ & 32,8716 & 0,9636 & $-22,9515$ \\
\hline 14 & $\mathrm{DG} \times \mathrm{GL} 2$ & 29,1949 & 0,9340 & $-31,5694$ \\
\hline 15 & $\mathrm{G} 2 \times \mathrm{GL} 1$ & 29,0074 & 0,8473 & $-32,0089$ \\
\hline 16 & $\mathrm{U} 1 \times \mathrm{GL} 2$ & 23,7040 & 0,9591 & $-44,4397$ \\
\hline 17 & C1×UGL & 15,6076 & 0,9645 & $-63,4170$ \\
\hline 18 & $\mathrm{C} \times \mathrm{C} 2$ & 11,7415 & 0,9655 & $-72,4788$ \\
\hline
\end{tabular}

Gisp (\%): ganho individual de seleção em relação à média geral das progênies $(42,6635)$ em porcentagem; u + g: valor genético predito.

A produtividade em plantas, em geral, está relacionada diretamente com maior grau de heterozigose, cuja expressão máxima ocorre em casos de hibridação interespecífica. Este fato pode ter contribuído para que a progênie DGxU2 tenha apresentado o maior valor genético (ODA; VENCOVSKY, 1989; SANTOS et al., 2016).

Os ganhos obtidos pela seleção entre progênies são inferiores ao de nível individual, uma vez que a seleção individual capitaliza 3/4 da variância de dominância enquanto a seleção entre progênies apenas $1 / 4$. Desta maneira, a seleção em nível individual feita via metodologia de modelos mistos apresenta propriedade ótima e maximiza os ganhos com seleção.

Realizar a seleção de toda uma progênie pode ocasionar o cruzamento de indivíduos aparentados, resultando na perda de alelos favoráveis e na diminuição do vigor devido a endogamia (BERTI et al., 2011). Dessa forma, se faz necessário realizar a otimização de seleção, que visa maximizar a diversidade genética na população, reduzir a endogamia e maximizar os ganhos genéticos para o próximo ciclo seletivo. Na otimização de seleção genética, seleciona-se as melhores progênies e os melhores indivíduos dentro de cada progênie, com o objetivo de maximizar o ganho genético e minimizar o coeficiente de endogamia.

\section{Otimização de seleção via tamanho efetivo populacional}

O processo de otimização de seleção da população simulou 30 diferentes cenários, com ganhos corrigidos variando de 97 a $170 \%$ e taxas de endogamia entre 3,7 a 25,8\% para o IMA (Tabela 8). Após a análise de cada situação, o melhor cenário ( $\left.N^{\circ} 19\right)$ foi escolhido com base no ganho corrigido e na endogamia.

A seleção de 10 indivíduos das 7 melhores progênies (Figura 1) resultou no cenário com ganho corrigido de 114,53\% e F no valor de 3,92\% (redução de 21,91\% em relação ao primeiro cenário).

Nos 5 primeiros cenários observou-se valores altos para o ganho corrigido, entretanto o tamanho efetivo populacional foi baixo, refletindo em alta porcentagem de endogamia (> 10\%). No decorrer das situações, houve a redução do ganho corrigido, de F e o aumento de Ne até a situação $\mathrm{N}^{\circ} 19$ (7 melhores progênies com 10 indivíduos cada). A seleção de indivíduos transgressivos resulta em maiores ganhos. Todavia, é importante observar o grau de parentesco desses para evitar o cruzamento de indivíduos aparentados (PEREIRA; VENCOVSKY, 1988). Assim, o cenário escolhido foi o № 19 $(\mathrm{F}=3,92 \%$ e ganho corrigido $=114,53 \%)$, pois a partir dele o tamanho efetivo populacional e a endogamia tenderam a se estabilizar e o ganho não sofreu alterações significantes.

Este processo aplicado dentro das estratégias de melhoramento genético busca um balanço adequado do número de progênies e indivíduos por cruzamento (CAIXETA, 2003). Com isso, é 
Tabela 8. Representação de potenciais cenários após o processo de otimização de seleção de progênies de Eucalyptus para a variável IMA ( $\mathrm{m}^{3}$.ha-1.ano) aos 6 anos de idade.

Table 8. Potential scenarios after the optimization process of Eucalyptus progenies selection for the trait MAI $\left(\mathrm{m}^{3} \cdot \mathrm{ha}^{-1} \cdot \mathrm{yr}\right)$ at age 6 years.

\begin{tabular}{|c|c|c|c|c|c|c|}
\hline $\mathbf{N}^{\circ}$ & Cenários & $\mathrm{Ne}$ & $\mathbf{F}$ & $F(\%)$ & Ganho (\%) & $\begin{array}{c}\text { Ganho } \\
\text { Corrigido } \\
(\%)\end{array}$ \\
\hline 1 & Melhor família - 30 ind & 1,9355 & 0,2583 & 25,8333 & 199,23 & 147,76 \\
\hline 2 & 2 Melhores Famílias - 3 ind & 3,0000 & 0,1667 & 16,6667 & 205,03 & 170,86 \\
\hline 3 & 2 Melhores Famílias - 5 ind & 3,3333 & 0,1500 & 15,0000 & 200,59 & 170,51 \\
\hline 4 & 2 Melhores Famílias - 10 ind & 3,6364 & 0,1375 & 13,7500 & 190,20 & 164,04 \\
\hline 5 & 3 Melhores Famílias - 3 ind & 4,5000 & 0,1111 & 11,1111 & 183,48 & 163,10 \\
\hline 6 & 3 Melhores Famílias - 5 ind & 5,0000 & 0,1000 & 10,0000 & 179,82 & 161,84 \\
\hline 7 & 3 Melhores Famílias - 10 ind & 5,4545 & 0,0917 & 9,1667 & 171,23 & 155,54 \\
\hline 8 & 4 Melhores Famílias - 3 ind & 6,0000 & 0,0833 & 8,3333 & 159,14 & 145,88 \\
\hline 9 & 4 Melhores Famílias - 5 ind & 6,6667 & 0,0750 & 7,5000 & 155,45 & 143,79 \\
\hline 10 & 4 Melhores Famílias - 10 ind & 7,2727 & 0,0688 & 6,8750 & 147,03 & 136,93 \\
\hline 11 & 5 Melhores Famílias - 3 ind & 7,5000 & 0,0667 & 6,6667 & 154,00 & 143,74 \\
\hline 12 & 5 Melhores Famílias - 5 ind & 8,3333 & 0,0600 & 6,0000 & 149,83 & 140,84 \\
\hline 13 & 5 Melhores Famílias - 10 ind & 9,0909 & 0,0550 & 5,5000 & 140,81 & 133,07 \\
\hline 14 & 6 Melhores Famílias - 3 ind & 9,0000 & 0,0556 & 5,5556 & 141,79 & 133,91 \\
\hline 15 & 6 Melhores Famílias - 5 ind & 10,0000 & 0,0500 & 5,0000 & 138,01 & 131,11 \\
\hline 16 & 6 Melhores Famílias - 10 ind & 10,9091 & 0,0458 & 4,5833 & 129,81 & 123,86 \\
\hline 17 & 7 Melhores Famílias - 3 ind & 10,5000 & 0,0476 & 4,7619 & 130,89 & 124,65 \\
\hline 18 & 7 Melhores Famílias - 5 ind & 11,6667 & 0,0429 & 4,2857 & 127,08 & 121,64 \\
\hline 19 & 7 Melhores Famílias - 10 ind & 12,7273 & 0,0393 & 3,9286 & 119,21 & 114,53 \\
\hline 20 & 7 Melhores Famílias - 15 ind & 13,1250 & 0,0381 & 3,8095 & 113,79 & 109,46 \\
\hline 21 & 7 Melhores Famílias - 20 ind & 13,3333 & 0,0375 & 3,7500 & 108,94 & 104,85 \\
\hline 22 & 7 Melhores Famílias - 25 ind & 13,4615 & 0,0371 & 3,7143 & 104,67 & 100,78 \\
\hline 23 & 7 Melhores Famílias - 30 ind & 13,5484 & 0,0369 & 3,6905 & 100,97 & 97,24 \\
\hline
\end{tabular}

Ne: Tamanho efetivo populacional; F: Coeficiente de endogamia; F(\%): Coeficiente de endogamia em porcentagem.

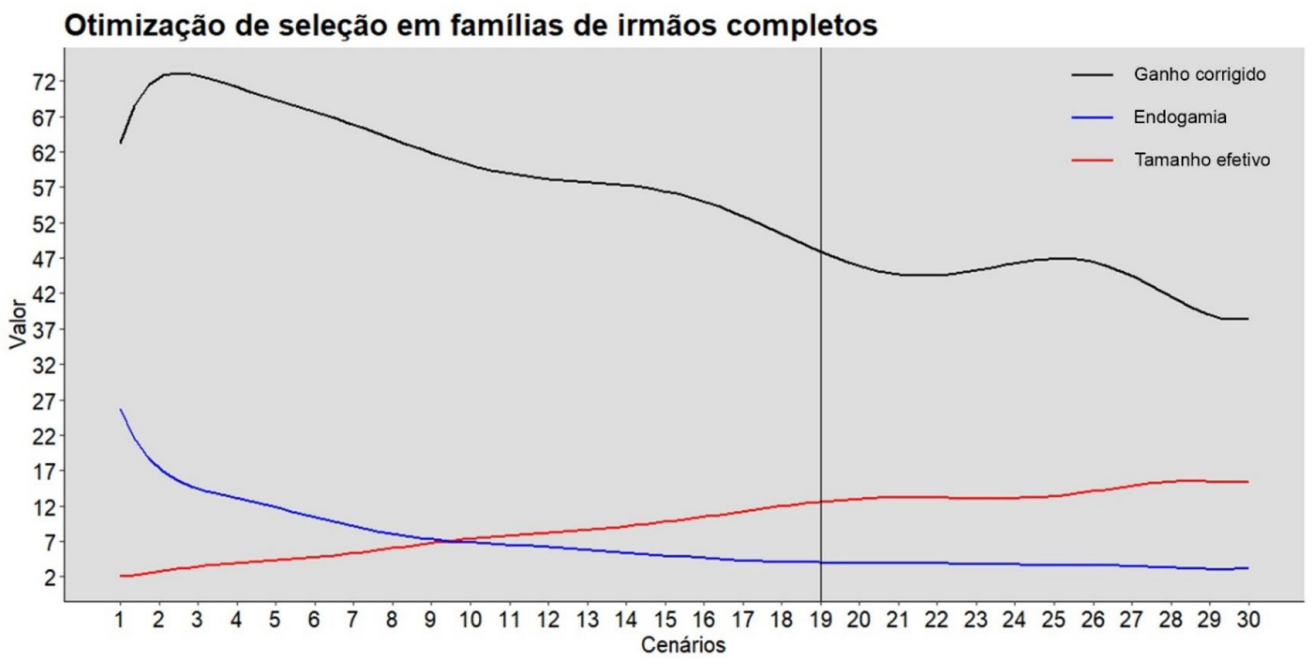

Figura 1. Otimização de seleção de progênies de irmãos completos de Eucalyptus a partir da simulação de 30 diferentes cenários, nos quais foram testados diferentes números de progênies selecionadas e de indivíduos dentro de cada progênie. A linha vertical no gráfico indica o cenário escolhido (19).

Figure 1. Optimization of Eucalyptus full-sib progenies selection through the simulation of 30 different scenarios, in which different numbers of selected progenies and individuals within each progeny were tested. The vertical line in the graph indicates the chosen scenario (19).

possível manter uma variabilidade genética relativamente alta e reduzir os problemas causados pela endogamia como produção de sementes não viáveis, geração de plantas anãs improdutivas e esgotamento da variância genética (HALLANDER; WALDMAN, 2009).

Para que se possa mensurar a representatividade genética de uma população avalia-se o tamanho efetivo populacional ( $\mathrm{Ne}$ ), o qual depende do número de progênies e indivíduos selecionados por 
Nogueira et al. - Estimativa de parâmetros genéticos em progênies de irmãos

completos de eucalipto e otimização de seleção

progênie. Ne está relacionado ao tamanho genético da população e não ao número de indivíduos que a compõe (FALCONER, 1960). Em situações de Ne baixo, poucos indivíduos participam efetivamente da geração de intercruzamentos, levando à ocorrência de dois eventos: mudanças aleatórias das frequências gênicas (oscilação genética) e aumento da endogamia na geração subsequente. Dessa maneira, o Ne é um parâmetro importante para conhecimento da perda de diversidade genética e depressão endogâmica. Para espécies de ciclo longo como o eucalipto, é crucial o uso eficiente de estratégias de seleção as quais possibilitem a maximização do Ne e minimização da endogamia (VALENTE et al., 2016). Com isso, torna-se essencial o controle do tamanho efetivo populacional na passagem de gerações.

Os resultados corroboram com os encontrados por Rocha et al. (2007) e Souza et al. (2011), que apresentaram ganhos significativos para a variável diâmetro à altura do peito (DAP) após o uso do tamanho efetivo populacional na realização da otimização de seleção em populações de meios-irmãos de eucalipto. Dessa maneira, é possível garantir a variabilidade genética das gerações futuras de melhoramento a partir dessa população experimental.

\section{CONCLUSÕES}

Os parâmetros genéticos estimados para as progênies de irmãos completos de eucalipto indicam condições favoráveis à seleção que, em associação com a otimização, podem gerar avanços genéticos consideráveis. Para proporcionar a maximização dos ganhos genéticos e a redução da endogamia, é recomendável que ocorra a seleção dos 10 melhores indivíduos presentes dentro das 7 melhores progênies.

A otimização de seleção é uma ferramenta que pode ser aplicada dentro dos programas de melhoramento e que possibilita maximizar a diversidade genética na população, reduzir a endogamia e maximizar os ganhos genéticos para o próximo ciclo seletivo.

\section{REFERÊNCIAS BIBLIOGRÁFICAS}

ALLARD, R. W. Princípios do melhoramento genético das plantas. São Paulo: Edgard Blucher, 1971, p. 381.

ASSIS, T. F. Melhoramento genético de Eucalyptus: desafios e perspectivas. 2014. In: ENCONTRO BRASILEIRO DE SILVICULTURA. 3., 2014, Campinas. Anais... Curitiba: Malinoviski, 2014.

ASSIS, T. F; MAFIA, R. G. Hibridação e clonagem. In: BORÉM, A. (Ed.). Biotecnologia florestal. Viçosa: Suprema, 2007, p. 93-121.

BERTI, C. L. F.; FREITAS, M. L. M.; ZANATTO, A. C. S.; MORAES, E.; SEBBENN, A. M. Variação genética, herdabilidades e ganhos na seleção para caracteres de crescimento e forma em teste de progênies de polinização aberta de Eucalyptus cloeziana. Revista Instituto Florestal, v. 23, n. 1, p. 13-26, 2011.

BISON, O.; RAMALHO, M. A. P.; REZENDE, G. D. S. P.; AGUIAR, A. M.; RESENDE, M. D. V. Dialelo parcial entre clones de Eucalyptus camaldulensis e clones de E. urophylla, E. grandis e E. saligna. Revista Árvore, v. 33, n. 3, p. 395-402, 2009.

BISON, O.; RAMALHO, M. A. P.; REZENDE, G. D. S. P.; AGUIAR, A. M.; RESENDE, M. D. V. Comparison Between Open Pollinated Progenies and Hybrids Performance in Eucalyptus grandis and Eucalyptus urophylla. Silvae Genetica, v. 55, n. 4-5, p. 192-196, 2006.

CAIXETA, R. P.; CARVALHO, D.; ROSADO, S. C. S. TRUGILHO, P. F. Variações genéticas em populações de Eucalyptus spp. detectadas por meio de marcadores moleculares. Revista Árvore, v. 27, n. 3, p. 357-363, 2003.

ESTOPA, R. A.; RAMALHO, M. A. P.; REZENDE, G. D. S. P.; ABAD, J. I. M.; GONÇALVES. Desempenho dos descendentes de clones de Eucalyptus spp. autofecundados e cruzados. Revista Cerne, v. 13, n. 3, p. 264-270, 2007.

FALCONER. D. S. Introduction to quantitative genetics. New York: Ronald Press, 1960, p. 365.

HALLANDER, J.; WALDMANN, P. Optimum contribution selection in large general tree breeding populations with an application to Scots pine. Theoretical and applied genetics, v. 118, n. 6, p. 1133-1142, 2009. 
HODGE, G. R.; VOLJER, P. W.; POTTS, B. M.; OWEN, J. V. A comparison of genetic information from openpollinated and control-pollinated progeny tests in two eucalypt species. Theoretical and Applied Genetics, v. 92, n. 1 , p. 53-63, 1996.

IBÁ - INDÚSTRIA BRASILEIRA DE ÁRVORES. Relatório anual: ano base 2016. Brasília: IBA, 2017.

KAGEYAMA, P. Y.; VENCOVSKY, R. Variação genética em progênies de uma população de Eucalyptus grandis (Hill) Maiden. IPEF, v. 24, p. 926, 1983.

LI, Y.; DUTKOWSKI, G. W; APIOLAZA, L. A; PILBEAM, D.; COSTA E SILVA, J; POTTS, B. M. The genetic architecture of a Eucalyptus globulus full-sib breeding population in Australia. Forest Genetics, v. 12, n. 3, p. 167-179, 2007.

LIMA, J. L.; SOUZA, J. C.; RAMALHO, M. A. P.; ANDRADE, H. B.; SOUSA, L. C. Early selection of parents and trees in Eucalyptus full-sib progeny tests. Crop Breeding and Applied Biotechnology, v. 11, n. 1, p. 10-16, 2011.

MIRANDA, A. C.; MORAES, M. L. T.; SILVA, P. H. M.; SEBBENN, A. M. Ganhos genéticos na seleção pelo método do índice multi-efeitos em progênies polinização livre de Eucalyptus grandis Hill ex Maiden. Scientia Forestalis, v. 43, n. 105, p. 203-209, 2015.

NUNES, A. C. P.; SANTOS, G. A.; RESENDE, M. D. V.; SILVA, L. D.; HIGA, A. R.; ASSIS, T. F. Estabelecimento de zonas de melhoramento para clones de eucalipto no Rio Grande do Sul. Scientia Forestalis, v. 44, n. 111 p. 563-574, 2016.

ODA, S.; MENCK, A. L. M.; VENCOVSKY, R. Problemas no melhoramento genético clássico do Eucalipto em função da alta intensidade de seleção. IPEF, n. 41-42, p. 8-17, 1989.

PEREIRA, M. B.; VENCOVSKY, R. Limites da seleção recorrente. I. Fatores que afetam o acréscimo das frequências alélicas. Pesquisa Agropecuária Brasileira, v. 23, n. 7, p. 769-780, 1988.

PEREIRA, R. C.; DAVIDE, L. C.; RAMALHO, M. A. P.; ANDRADE, H. B. Alternatives to improve hybridization efficiency in eucalyptus breeding programs. Revista Cerne, v. 8, n. 2, p. 60-69, 2015.

PINTO, D. S.; RESENDE, R. T.; MESQUITA, A. G. G.; ROSADO, A. M.; CRUZ, C. D. Seleção precoce para características de crescimento em testes clonais de Eucalyptus urophylla. Scientia Forestalis, v. 42, n. 102, p. 251-257, 2014.

REIS, C. A. F.; GONÇALVES, F. M. A.; RAMALHO, M. A. P.; ROSADO, A. M. et al. Seleção de progênies de eucalipto pelo índice Z por MQM e Blup. Pesquisa agropecuária brasileira, v. 46, n. 5, p. 517-523, 2011.

RESENDE, M. D. V. Software Selegen-REML/BLUP: a useful tool for plant breeding. Crop Breeding and Applied Biotechnology, v. 16, n. 4, p. 330-339, 2016.

RESENDE, M. D. V. Genética Biométrica e Estatística no Melhoramento de Plantas Perenes. Brasília: Embrapa informações tecnológicas, 2002, 975 p.

RESENDE, M. D. V. Melhoramento de essências florestais. In: BORÉM, A. Melhoramento de espécies cultivadas. Viçosa: Editora UFV, 1999. p. 589-647.

RESENDE, M. D. V. Delineamento de experimentos de seleção para maximização da acurácia seletiva e do progresso genético. Revista árvore, v. 19, n. 4, p. 479-500, 1995.

RESENDE, M. D. V.; DUARTE, J. B. Precisão e controle de qualidade em experimentos de avaliação de cultivares. Pesquisa Agropecuária Tropical, v. 37, n. 3, p. 182-194, 2007.

RESENDE, M. D. V.; HIGA, A. R. Estimação de valores genéticos no melhoramento de Eucalyptus: seleção em um caráter com base em informações do indivíduo e de seus parentes. Boletim de Pesquisa Florestal, $\mathrm{n}$. 28-29, p. 11-36, 1994.

RESENDE, M.D.V.; HIGA, A. R. Estratégias de melhoramento para Eucalyptus visando à seleção de híbridos. Boletim de Pesquisa Florestal, v. 21, n. 1, p. 49-60, 1990.

ROCHA, M. G. B.; PIRES, I. E.; ROCHA, R. B.; XAVIER, A.; CRUZ, C. D. Seleção de genitores de Eucalyptus grandis e de Eucalyptus urophylla para produção de híbridos interespecíficos utilizando REML/BLUP e informação de divergência genética. Revista Árvore, v. 31, n. 6, p. 977-987, 2007. 
Nogueira et al. - Estimativa de parâmetros genéticos em progênies de irmãos

completos de eucalipto e otimização de seleção

ROSADO, A. M.; ATAPIDE, G. M.; CASTRO, R. V. O.; CORREIA, A. C. G. Avaliação da tolerância à quebra por vento em árvores de eucalipto via teste de resistência. Pesquisa Florestal Brasileira, v. 33, n. 75, p. 309-315, 2013.

SANTOS, G. A.; NUNES, A. C. P.; RESENDE, M. D. V.; SILVA, L. D.; HIGA, A.; ASSIS, T. F. An index combining volume and Pilodyn penetration to study stability and adaptability of Eucalyptus multi-species hybrids in Rio Grande do Sul, Brazil. Australian Forestry, v. 79, n. 4, p. 248-255, 2016.

SANTOS, G. A.; RESENDE, M. D. V.; SILVA, L. D.; HIGA, A.; ASSIS, T. F. Adaptabilidade de híbridos multiespécies de Eucalyptus ao Estado do Rio Grande do Sul. Revista Árvore, v. 37, n. 4, 2013.

SCHUMACHER, F. X.; HALL, F. S. Logarithmic expression of timber-tree volume. Journal of Agricultural Research, v. 47, n. 9, p. 719-734, 1933.

SILVA, J. C.; BORALHO, N. M.; POTS, B. M. Additive and non-additive genetic parameters from clonally replicated and seedling progenies of Eucalyptus globulus. Theoretical and Applied Genetics, v. 108, n. 6, p. 1113-1119, 2004.

SOUZA, F. M. L.; PUPO, C. H.; SEREGHETTI, G. C.; SANSÍGOLO, C. A.; FERREIRA, J. P.; SILVA, R. B.; GARCIA, D. P. Características de crescimento, densidade básica e composição química da madeira de Eucalyptus spp na região de Ribas do Rio Pardo - MS. Revista Brasileira de Engenharia de Biossistemas, v. 11, n. 4, p. 350359, 2017.

SOUZA, C. S.; FREITAS, M. L. M.; MORAES, M. L. T.; SEBBENN, A. M. Estimativas de parâmetros genéticos para caracteres quantitativos em progênies de polinização aberta de Eucalyptus urophylla. Floresta, v. 41, n. 4, p. 847-856, 2011.

VALENTE, M. S. F.; VIANA, J. M. S.; RESENDE, M. D.; SILVA, F. F.; LOPES, M. T. G. Seleção genômica para melhoramento vegetal com diferentes estruturas populacionais. Pesquisa Agropecuária Brasileira, v. 51, n. 11, p. 1857-1867, 2016.

VENCOVSKY, R.; BARRIGA, P. Genética biométrica no fitomelhoramento. Ribeirão Preto: Sociedade Brasileira de Genética, 1992. 496 p.

WOOLLIAMS, J. A.; BERG, P.; DAGNECHEW, B. S.; MEUWISSEN, T. H. E. Genetic contributions and their optimization. Journal of Animal Breeding and Genetics, v. 132, n. 2, p. 89-99, 2015.

Recebido em: 03/10/2017

Aceito em: 13/11/2018 